\title{
Extraperitoneal versus transperitoneal cesarean section: a retrospective analysis
}

\author{
Babita P. Vaswani, Aditi Trivedi*, Sriram Gopal
}

Department of Obstetrics and Gynecology, Dr D. Y. Patil Hospital, Nerul, Navi Mumbai, Maharashtra, India

Received: 10 May 2019

Revised: 30 December 2019

Accepted: 02 January 2020

\section{*Correspondence:}

Dr. Aditi Trivedi,

E-mail: aditi.trivedi@yahoo.in

Copyright: (c) the author(s), publisher and licensee Medip Academy. This is an open-access article distributed under the terms of the Creative Commons Attribution Non-Commercial License, which permits unrestricted non-commercial use, distribution, and reproduction in any medium, provided the original work is properly cited.

\begin{abstract}
Background: Cesarean section is becoming a more common delivery type worldwide. The objective of this study was to compare surgical morbidity in extraperitoneal versus transperitoneal techniques of cesarean section.

Methods: A total of 60 patients was included in this study out of which 30 underwent extraperitoneal cesarean section(ECS) and 30 patients underwent transperitoneal cesarean section (TCS) and compared both the techniques with respect to nausea or vomiting intra-operatively, pain score (as measured by visual analogue scale) post operatively, return of bowel function and blood loss.

Results: Intra-operative nausea and vomiting was noted in $33.34 \%$ of patients in TCS group while none of the patients in ECS group had any such complaints. No difference was noted in average blood loss between the 2 groups. Post-operative pain in ECS was 4.13 as compared to 6.86 in TCS. Return of bowel sounds in ECS group was much earlier (5.46 hours) as compared to TCS group (11.33 hours).

Conclusions: We conclude that extraperitoneal technique is advantageous over transperitoneal approach. Decreased nausea and vomiting, early return of bowel function in the post-operative period, decreased post-operative pain allowing early feeding and mobilization are definite benefits of this technique. This further helps in making a relatively smoother post-operative recovery.
\end{abstract}

Keywords: Cesarean section, Extraperitoneal, Intraperitoneal, Surgical morbidity

\section{INTRODUCTION}

Cesarean section is becoming a more common delivery type worldwide. In our institute, Dr D. Y. Patil Hospital, rate of cesarean sections is $46.17 \%$ which is higher than WHO recommended cut off $(15 \%)$ as ours is a tertiary care referral centre. ${ }^{1}$ It is still a major surgical procedure that carries risks for both mother and child. As the incidence of cesarean sections is increasing, infective complications related to it are also on the rise. Avoidance of the peritoneal cavity is surgically sound and is a primary defence against infections that complicate cesarean sections. ${ }^{2}$ Extraperitoneal approach is a method of least invasiveness and hence it is a safer alternative to the traditional transperitoneal technique. Traditionally, transperitoneal cesarean section includes opening the parietal peritoneum and delivering the baby through a transverse incision in the lower uterine segment. ${ }^{3}$

Extraperitoneal cesarean section is the method in which the baby is delivered by making an incision in the lower segment of the uterus which is approached through the paravesical space without entering peritoneal cavity. It proves to be beneficial in cases like prolonged labour, ruptured membranes over 24 hours, repeated vaginal examinations in which there is fear of spreading infection 
from the uterus to the peritoneal cavity. Extraperitoneal approach was presumed to reduce postoperative intraabdominal infections and also nausea and vomiting, postoperative pain by avoiding exposure of the peritoneal cavity to blood, amniotic fluid, vernix, and mechanical irritation. ${ }^{4}$

With advent of antibiotic era, transperitoneal technique has become the approach of choice. However, as antibiotic resistance has become a reality and cesarean delivery becoming a common procedure, extraperitoneal approach may prove to be more beneficial in avoiding infections, reducing morbidity and making post-operative recovery faster and smoother for the mothers. So, it has now become imperative that practicing obstetricians find ways to reduce perioperative and post-operative morbidity. It is in this sense that extraperitoneal cesarean section gets its true outlook as it represents a viable alternative to the transperitoneal technique. ${ }^{5}$

\section{METHODS}

This study was conducted in the obstetrics and gynecology department of Dr D. Y. Patil Hospital and Medical Research Centre, Navi Mumbai for a period of 6 months between January 2019 to June 2019. A total of 60 patients were included in this study.

\section{Inclusion criteria}

- All those without the above-mentioned conditions.

\section{Exclusion criteria}

- Abnormal placentation

- Non vertex presentation

- Previous LSCS

- Previous major abdominal surgery

- Preterm

- Fetal distress

- Antepartum hemorrhage.

Included patients were put into 2 groups equally by alternate randomization - Group A (extraperitoneal cesarean section - ECS) and Group B (transperitoneal cesarean section - TCS). Written valid informed consent was taken and Foley's catheter was placed preoperatively for all patients.

Bupivacaine was given for spinal anesthesia as a uniform protocol for patients in both the groups. $3^{\text {rd }}$ generation cephalosporin was administered intravenously half an hour prior to skin incision in all patients.

Pfannenstiel incision was taken in both cases.

In ECS, abdomen was opened in layers up to the rectus muscle. The recti are separated adequately and pre peritoneal dissection is done. The median umbilical ligament is identified and pushed medially. Bladder is pushed infero-laterally and an incision is made in the lower uterine segment and baby delivered. Instrumentation was used whenever necessary. Uterine incision is closed in single layer. After confirming hemostasis, abdomen was closed in layers.

TCS is performed in the conventional manner. Uterus was not exteriorized for suturing. Parietal peritoneum was closed.

Skin was closed by subcuticular sutures with Monocryl in both groups.

Intra op nausea and vomiting, blood loss was recorded.

Post operatively, intravenous NSAIDS were given 8 hourly to all patients. Postoperative pain at 6 hours post operation was calculated by visual analogue scale. For those requiring more analgesia, opioid analgesics were added. Bowel sounds were checked every 2 hours and return of bowel function was noted accordingly. Oral feeds were started according to the return of bowel functions. Patients were discharged based on passage of stools and no other complaints.

The above-mentioned parameters were then compared between the 2 groups.

\section{Statistical analysis}

Method of statistical analysis includes averages and percentages.

\section{RESULTS}

It was seen that no patient in ECS group complained of intra operative nausea, vomiting whereas 10 patients $(33.34 \%)$ developed nausea in TCS group intra operatively. Average blood loss in ECS and TCS was 476 $\mathrm{ml}$ and $490 \mathrm{ml}$ respectively. In our study, return of bowel sounds was noted at 5.46 hours in ECS group which is much earlier as compared to $11.33 \mathrm{hrs}$ in TCS group. Post op pain, as calculated by VAS score, was 4.13 in ECS group and 6.86 in TCS group (Table 1). There were no intra-operative complications in either of the groups.

Table 1: Comparison of various parameters between the two groups.

\begin{tabular}{|lll|}
\hline Parameters & ECS & TCS \\
\hline Intra op nausea, vomiting & $0 \%$ & $33.34 \%$ \\
\hline Return of bowel sounds & 5.46 hours & 11.33 hours \\
\hline Blood loss & $476 \mathrm{ml}$ & $490 \mathrm{ml}$ \\
\hline Post op pain & 4.13 & 6.86 \\
\hline
\end{tabular}

\section{DISCUSSION}

Total of 60 cases were included in the study. Out of which, 30 were extraperitoneal sections (ECS) and 30 were transperitoneal sections (TCS). There was no 
significant difference in the blood loss between both the groups. As peritoneum is intact in ECS, there is minimal bowel irritation which leads to early return of bowel sounds. This in turn, facilitated in starting oral fluids earlier in ECS group. Moreover, the need for analgesic in ECS group was also lesser as compared to TCS group. Similar observations were made in a study done by Zabransky et al and Yesilbas et al. ${ }^{3,6}$ Also, the finding that no patient in ECS group developed nausea and vomitting is consistent with the study done by Bebincy, et al and Carmen et al. ${ }^{4,7}$

Less pain with early feeding helped in early mobilization of the patients in ECS group along with early catheter removal allowing a smooth post-operative recovery. This was in consistence with a study done by Wallace $\mathrm{R}$ et al, where there was a trend toward enhanced postoperative recovery in all extraperitoneal cesarean section groups compared with the transperitoneal primary cesarean section control group. ${ }^{8}$ Also, all this led to reduced hospital stay in ECS group which proved to be beneficial economically for patients in ECS group.

\section{CONCLUSION}

Extraperitoneal technique is definitely advantageous over transperitoneal approach. Decreased nausea and vomiting, decreased post-operative pain, early return of bowel function in the post-operative period allowing early feeding and mobilization are the benefits of this technique. The above-mentioned advantages allow enhanced post-operative recovery in ECS thus giving ECS the stand point it deserves. Moreover, avoidance of entry in to the peritoneal cavity helps to prevent potential intraperitoneal bowel and bladder adhesions and decrease the difficulty of subsequent laparotomy or laparoscopy.

Therefore, this technique should be taught and encouraged amongst practicing obstetricians as a means to reduce surgical morbidity in cesarean sections and subsequent abdominal surgeries.
Funding: No funding sources

Conflict of interest: None declared

Ethical approval: Not required

\section{REFERENCES}

1. Gandi SR, Vaswani B, Gopal S. Analysis of rates of cesarean sections using Robson's 10- group classification in a tertiary care hospital. MedPulse Int J Gynecol. 2019;10(2):87-90.

2. Hanson HB. Current use of the extraperitoneal cesarean section: a decade of experience. Am J Obstet Gynecol. 1984;149(1):31-4.

3. Zabransky F, Grossmanova H. Extraperitoneal cesarean section-an alternative or routine?. Ceska Gynecol. 2001;66(3):187-9.

4. Bebincy DS, Chitra J. Extraperitoneal versus transperitoneal cesarean section in surgical morbidity in a tertiary care centre. Int J Reprod Contracept Obstet Gynecol. 2017;6:3397-9.

5. Imig JR, Perkins RP. Extraperitoneal cesarean section; a new need for old skills. A preliminary report. Am J Obslet Gyrecol. 1976;125(1):51-4.

6. Yesilbas C, Erenel H. Extraperitoneal versus transperitoneal cesarean section: a retrospective analysis. Perinatal J. 2017;25(1):38-42.

7. Tappauf C. Extraperitoneal versus transperitoneal cesarean section: a prospective randomized comparison of surgical morbidity. Am J Obstet Gynecol. 2013;209(4):338-e1.

8. Wallace RL, Eglington GS, Yonekura ML, Wallace TM. Extraperitoneal cesarean section: a surgical form of infection prophylaxis? Am J Obstet Gynecol. $1984 ; 148(2): 17-7$.

Cite this article as: Vaswani BP, Trivedi A, Gopal

$\mathrm{S}$. Extraperitoneal versus transperitoneal cesarean section: a retrospective analysis. Int J Reprod Contracept Obstet Gynecol 2020;9:567-9. 